\title{
Late-onset secondary nocturnal enuresis in adolescents associated with post-traumatic stress disorder developed after a traffic accident
}

\author{
Serkan Akan, Ahmet Ürkmez, Caglar Yildirim, Aytac Sahin, Özgür Haki Yüksel, Ayhan Verit \\ Fatih Sultan Mehmet Research \& Training Hospital, Dept. of Urology, Istanbul, Turkey.
}

\begin{abstract}
Summary Secondary nocturnal enuresis is generally seen between 5 and 7 years of age and it is rarely encountered when compared with the primary incontinence. Patients with suggested diagnosis of secondary nocturnal enuresis should be examined for neurological and spinal anomalies and diabetes mellitus, diabetes insipidus, renal failure and urinary tract infection should be ruled out in differential diagnosis (1-3). Herein, we are presenting case reports of adolescent patients with secondary nocturnal enuresis refractory to medical therapy and developed after in-vehicle and extravehicular accidents.
\end{abstract}

KEY WORDS: Secondary nocturnal enuresis; Medical therapy; Psychotherapy; Adolescent.

Submitted 8 May 2015; Accepted 30 June 2015

\section{INTRODUCTION}

Enuresis nocturna is defined as involuntary night-time bedwetting during sleep in children over 5 years of age. It is a very disturbing common problem for children and their families. However secondary nocturnal enuresis is generally seen between 5 and 7 years of age and it is quite rare compared with primary nocturnal enuresis. Herein, we are presenting case reports of adolescent patients with secondary nocturnal enuresis refractory to medical therapy and developed after in-vehicle and extravehicular accidents.

\section{Case presentations}

Case 1

A 14-year-old girl had an extravehicular traffic accident. The same day she was examined in the emergency service. Since she hadn't any serious wound(s), she was discharged without prescribing any medical therapy. She presented to us with complaints of nocturnal enuresis recurring nearly every night

The patient did not mention any behavioural problem starting after the traffic accident. She hadn't any complaint of bedwetting before the accident. Her physical examination findings were within physiologic limits. Any abnormality was not observed in her laboratory findings, urinalysis, urine culture and urinary system ultrasound. During 2 months of follow-up with prescription of keeping a voiding diary, behavioural treatment and fluid restriction, her complaints decreased, but still persisted. Then daily oral desmopression (one 120 $\mu \mathrm{g}$ lyophilized tablet a day) therapy was initiated. Since her complaints did not fully resolve, she was transferred to the department of pediatric psychiatry and complete cure was achieved with combined treatment.

\section{Case 2}

A 12-year-old girl had an in-vehicle accident nearly 6 months previously. At the time of the accident, a serious wound except for a minor head trauma was not observed in the patient sitting in the back seat and she was evaluated the same day in the emergency service and discharged. She consulted to us with complaints of night-time bedwetting (enuresis nocturna) persisting for 6 months. She expressed that from the day of the accident on she had complaints of sleeping disorders and she had been having nightmares. Before the accident she hadn't had any complaint of incontinence. Her physical and neurological examination findings were within normal limits. Laboratory parameters, urinalysis, urine culture and urinary system ultrasound findings were unremarkable.

The patient was started on daily oral dsesmopressin therapy (one $120 \mu \mathrm{g}$ lyophilized tablet a day). Her complaints regressed at a rate of 50 percent. Since complete dryness could not be achieved, she was consulted to pediatric psychiatry department.

She underwent psychotherapy in combination with medical therapy for 3 months and her complaints regressed considerably.

\section{Discussion}

Enuresis nocturna is a quite disturbing common problem for children and their families. The underlying causative factors are functional disorders as bladder dysfunction. However it can be rarely seen in association with anatomical abnormalities and neurogenital disorders and diseases as diabetes insipidus, chronic renal failure and tubulopathies. Besides, some cases with nocturnal enuresis have been observed after use of clozapine, risperidone and valproic acid (1). 
Its psychological etiology has not been known yet. However delayed maturation of cortical or central autonomic mechanisms regulating the functions of voluntary micturition center has been stated in its pathogenesis (2). Secondary nocturnal enuresis is generally seen between 5 and 7 years of age and it is rarely encountered when compared with the primary incontinence. Patients with suggested diagnosis of secondary nocturnal enuresis should be examined for neurological and spinal anomalies and diabetes mellitus, diabetes insipidus, renal failure and urinary tract infection should be ruled out in differential diagnosis.

The largest scale-study in the literature has been conducted by Tal Eidlitz-Markus et al. on 5 patients with secondary nocturnal enuresis. As is reported by us, classifical information about late-onset secondary nocturnal enuresis or secondary nocturnal enuresis in adolescents have not been cited in relevant references.

Incidence of incontinence is higher in children with behavioural and emotional problems (3). In some studies performed, the authors stated that $20-40 \%$ of the children with daytime and $20-30 \%$ of night-time urinary incontinence and $50 \%$ of pediatric cases with fecal incontinence met DSM-IV (Diagnostic and Statistical Manual of Mental Disorders) criteria of psychiatric disorders $(4,5)$. In these studies, the authors indicated that compliance with psychological treatment favourably contributed to success rates of the treatment of incontinence.

From the clinical perspective, the incidence of behavioural disorders has been indicated in 10-15\% of children and adolescents. These behavioural disorders include anxiety, fear, depressive mood, hyperactivity, anorexia nervosa and autism. In these patients incidence of incontinence (including all of its subtypes) is higher relative to normal continent population.

Epidemiological studies have demonstrated that $20-30 \%$ of the children with nocturnal enuresis displayed behavioural problems. Besides, these rates demonstrate increases in line with age, male gender and lower socioeconomic status (6). Subclinical behavioural problems may go undetected. Apparently all subtypes of incontinence constitute a risk for lower quality of life and loss of self-esteem (7). However, parents of these children become intolerant to this problem and put their children under a lot of stress (8). Literature studies have indicated that children diagnosed as nocturnal enuresis also carry higher (75\%) risk of psychological disorders (9).

Following posttraumatic stress disorders, symptoms of hyperactivity, decrease in attention span, bursts of anger, sleep disorders, distemper and undisciplined behaviours, headache and dizziness can be seen.

International Children's Continence Society (ICCS) has released psychological and psychiatric evaluation and treatment guidelines for pediatric patients with day- and night-time urinary or fecal incontinence (10).

It should not be forgotten that posttraumatic secondary nocturnal enuresis and incontinence can be seen during adolescent period. In addition to its specific treatment, pediatric psychological and psychiatric assessments in line with evidence-based application parametres and directives should be suggested. The importance of behavioural counselling and healthcare services in the acquisition of optimal therapeutical outcomes for the children and their families should be taken into consideration.

\section{Conclusions}

It should not be forgotten that posttraumatic secondary nocturnal enuresis and incontinence can be seen during adolescent period. In addition to its specific treatment, pediatric psychological and psychiatric assessments in line with evidence-based application parametres and directives should be suggested.

The importance of behavioural counselling and healthcare services in the acquisition of optimal therapeutical outcomes for the children and their families should be taken into consideration.

\section{REFERENCES}

1. Zaiem A, Aouinti I, Lakhoua G, et al. Secondary nocturnal enuresis associated with valproic acid. Therapie. 2013; 68:59-60.

2. Eidlitz-Markus T, Shuper A, Amir J. Secondary enuresis: posttraumatic stress disorder in children after car accidents. Isr Med Assoc J. 2000; 22:135-7.

3. Von Gontard A and Nevéus T: Management of Disorders of Bladder and Bowel Control in Childhood. London: MacKeith Press 2006.

4. World Health Organization: Multiaxial Classification of Child and Adolescent Psychiatric Disorders: The ICD-10 Classification of Mental and Behavioural Disorders in Children and Adolescents. Cambridge: Cambridge University Press 2008.

5. American Psychiatric Association: Diagnostic and Statistical Manual of Mental Disorders, Text Revision (DSM-IV-TR). Washington, DC 2000.

6. Baeyens D, Roeyers H, Vande Walle J, et al: Behavioural problems and attention-deficit hyperactivity disorder in children with enuresis: a literature review. Eur J Pediatr. 2005; 164:665.

7. Bachmann C, Lehr D, Janhsen E, et al. Health related quality of life of a tertiary referral center population with urinary incontinence using the DCGM-10 questionnaire. J Urol. 2009; 182:2000.

8. Butler RJ, Golding J, Heron J, et al: Nocturnal enuresis: a survey of parental coping strategies at 7 1/2 years. Child Care Health Dev. 2005; 31:659.

9. Von Gontard A, Mauer-Mucke K, Plück J, et al: Clinical behavioral problems in day- and nightwetting children. Pediatr Nephrol. 1999; 13:662.

10. Nevéus T, von Gontard A, Hoebeke P, et al. The standardization of terminology of lower urinary tract function in children and adolescents: report from the Standardisation Committee of the International Children's Continence Society. J Urol. 2006; 176:314.

\section{Correspondence \\ Serkan Akan, MD \\ Ahmet Ürkmez, MD \\ Caglar Yildirim, MD \\ Aytac Sahin, MD \\ Özgür Haki Yüksel, MD (Corresponding Author) ozgurhaki@gmail.com \\ Ayhan Verit, MD. Prof.}

Fatih Sultan Mehmet Research and Training Hospital, Dept. of Urology, Içerenköy/Ataşehir, Tr- 34752 Istanbul, Turkey 